\title{
Alterações dos óleos de palma e de soja em fritura descontínua de batatas
}

\author{
Palm and soybean oils alterations in the discontinued frying of potatoes
}

\author{
Eliana Rodrigues MACHADO ${ }^{1 *}$, María del Carmen Dobarganes GARCÍA ${ }^{2}$, Shirley de Mello Pereira ABRANTES ${ }^{1}$
}

\begin{abstract}
Resumo ser eficazmente verificada através da determinação quantitativa de polímeros.

Palavras-chave: ácidos graxos; óleos de fritura; óleo de palma; óleo de soja; polímeros.
\end{abstract}

Óleos de palma e de soja foram utilizados em experimentos de fritura de batatas em fritadeiras elétricas domésticas de $1 \mathrm{~L}$ com relação inicial, superfície sobre volume, de $0,3 \mathrm{~cm}^{-1}$. Em cinco dias consecutivos cada óleo foi submetido por um período de 5 horas por dia à temperatura de $(183,1 \pm 1,7)^{\circ} \mathrm{C}$. Após 25 horas, a perda de ácidos graxos foi de 15,0 e 62,2\% $(\mathrm{m} / \mathrm{m})$, respectivamente, para os ácidos oléico e linoléico, no óleo de palma; e de 6,5;24,9 e 39,7\% (m/m), respectivamente, para os ácidos oléico, linoléico e linolênico, no óleo de soja. Os teores inicial e final de polímeros foram de 0,4 e 23,7\% (m/m) no óleo de palma, e de 0,5 e 30,7\% (m/m) no óleo de soja. A relação entre a perda dos ácidos graxos e a formação de polímeros mostrou uma correlação forte com coeficientes de correlação de 0,9951 e 0,9740 para os óleos de palma e de soja, respectivamente. Concluiu-se que maiores graus de alteração ocorreram em ácidos graxos mais insaturados; e que a alteração pode

\begin{abstract}
Palm and soybean oils were used in experiments of frying of potatoes in $1 \mathrm{~L}$, household electric deep fryers with a surface-to-volume ratio of $0,3 \mathrm{~cm}^{-1}$. The oils were heated for five hours every day for five consecutive days at a temperature of $183.1 \pm 1.7^{\circ} \mathrm{C}$. the evaluation of oil degradation was followed by the increase of polymer contents and changes in fatty acid composition. After 25 hours, the loss of fatty acids were 15.0 and $62.2 \%$ for oleic and linoleic acid, respectively in palm oil and 6.5, 24.9 e $39.7 \%$ for oleic, linoleic, and linolenic acid, respectively in soybean oil. The content of polymers ranged from 0.4 to $23.7 \%$ and from 0.5 to $30.7 \%$ for palm and soybean oils, respectively. Linear association between the loss of total unsaturated fatty acids and polymers formation presented high correlation coefficients ( 0.9951 and 0.9740 for palm and soybean oils, respectively. The results obtained clearly indicate that the higher the degree of fatty acid unsaturation, the higher its degradation, and also that the quantitative determination of polymers would be a very useful analysis to evaluate the oil degradation.
\end{abstract}

Keywords: fatty acids; frying oils; palm oil; polymers; soybean oil.

\section{Introdução}

Diversos estudos têm concluído que substâncias, que podem ser formadas em óleos e gorduras usados em frituras, podem ser prejudiciais à saúde humana (DOBARGANES; MÁRQUEZ-RUIZ, 2003). Avaliações da qualidade de óleos e gorduras usados em frituras realizadas em diversos países, inclusive no Brasil, têm demonstrado a necessidade de mais estudos sobre o processo de fritura, pois foram encontradas porcentagens acima de $30 \%$ destes óleos e gorduras com níveis altos de alteração (CROON, et al., 1986; DOBARGANES; MÁRQUEZ-RUIZ, 1995; GERTZ, 1986; LAKE; SCHOLES, 1997; MATTOS; ANS; JORGE, 2000; SEBEDIO, et al., 1987; SKRÖKKI, 1995; VAHCIC; HRUSKAR, 1999). Nas avaliações destes estudos se utilizou a determinação quantitativa de compostos polares, parâmetro mais recomendado por legislações internacionais para verificar o nível de alteração dos óleos e gorduras em uso em frituras (DANA; SAM SAGUY, 2001).
Os óleos e gorduras usados em frituras são submetidos a altas temperaturas em presença de ar e água. Esta condição propicia a formação de um número considerável de substâncias oriundas de reações térmicas, oxidativas e hidrolíticas (CHANG; PETERSON; HO, 1978; DOBARGANES; PÉREZ-CAMINO, MÁRQUEZ-RUÍZ, 1998; FRITSCH, 1981; GASPAROLI, 1998; JORGE et al., 1996; MÁRQUEZ-RUÍZ; TASIOULA-MARGARI; DOBARGANES, 1995; NAWAR, 1998; SCHWARTZ; RADY; CASTAÑEDA, 1994; VELASCO et al., 2004a; 2004b). Estas reações resultam na formação de uma mistura complexa de substâncias conhecida como "compostos polares" (DOBARGANES; PÉREZ-CAMINO; MÁRQUEZ-RUIZ, 1988). As reações hidrolíticas ocorrem nos triacilgliceróis, constituintes majoritários dos óleos e gorduras, liberando mono e diacilgliceróis, ácidos graxos livres e glicerol. As reações térmicas e oxidativas, que ocorrem prioritariamente nas ligações duplas dos ácidos graxos

Recebido para publicação em 24/5/2007

Aceito para publicação em 14/10/2008 (002537)

${ }^{1}$ Laboratório Alimentos e Contaminantes, Departamento de Química, Instituto Nacional de Controle de Qualidade em Saúde, Fundação Oswaldo Cruz, CEP 21040-900,

Rio de Janeiro - RJ, Brasil, E-mail: eliana.machado@incqs.fiocruz.br

2 Departamento de Caracterización y Calidad de los Alimentos, Caracterización y Modificación de Grasas y Aceites, Instituto de la Grasa,

Consejo Superior de Investigaciones Científicas, CEP 41012, Sevilha, Espanha

${ }^{*}$ A quem a correspondência deve ser enviada 
livres ou esterificados à molécula de glicerol, como mono, di e triacilgliceróis, formam principalmente as substâncias dos grupos dos monômeros de triacilgliceróis oxidados (monômeros oxidados) e dos triacilgliceróis dimerizados e polimerizados (dímeros e polímeros). Estas reações também podem levar à degradação dos ácidos graxos, livres e ligados, formando aldeídos, cetonas, substâncias cíclicas dentre outras (FRITSCH, 1981). Então, as alterações que podem ocorrer nos ácidos graxos em processos de fritura resultam de reações térmicas e oxidativas com a conseqüente perda destes ácidos.

Em óleos e gorduras que foram usados em frituras e com níveis significativos de alteração, verificou-se que o grupo dos dímeros e polímeros é formado em maiores proporções do que o grupo dos monômeros oxidados (MÁRQUEZ-RUÍZ; TASIOULA-MARGARI; DOBARGANES, 1995). Outros estudos também demonstraram maior formação de polímeros em relação aos monômeros oxidados, quando consideráveis níveis de degradação das amostras estavam presentes: em sistemas modelo de ésteres metílicos dos ácidos oléico e linoléico termoxidados a $180^{\circ} \mathrm{C}$ (BERDEAUX; MÁRQUEZ-RUÍZ; DOBARGANES, 1999); em óleos de oliva e de girassol termoxidados a $180^{\circ} \mathrm{C}$; e em óleos e gorduras usados em fritura (VELASCO et al., 2004a).

O nível de alteração dos ácidos graxos depende de muitas variáveis, dentre elas: temperatura; tempo; aquecimento descontínuo ou contínuo; grau de mudança do meio de fritura, reposição ou substituição por óleo novo; etc. Nos processos descontínuos de fritura, utilizados em residências, restaurantes, lanchonetes, a alteração do óleo ou gordura é alta e geralmente é devido à ocorrência de ciclos de aquecimento e resfriamento e ao baixo grau de mudança do meio de fritura (DOBARGANES; MÁRQUEZ-RUIZ, 1998).

O grau de insaturação do óleo ou gordura é também uma variável importante no processo de fritura (DOBARGANES; MÁRQUEZ-RUIZ, 1998). E por isso, foram escolhidos, para este estudo, os óleos de palma e de soja que apresentam distintos graus de insaturação, um com predominância de ácidos graxos poliinsaturados (óleo de soja) e outro com predominância de ácidos graxos monoinsaturados e saturados (óleo de palma) (FORMO, 1979).

O objetivo deste estudo foi conhecer alterações termoxidativas, através da perda de ácidos graxos e da formação de polímeros, nos óleos de palma e de soja, em frituras descontínuas de batatas.

\section{Material e métodos}

\subsection{Amostra inicial - antes da utilização no experimento}

O óleo de palma refinado comercializado para fritura foi obtido por doação da indústria "Grupo Agropalma" da cidade de Tailândia, Pará, com certificado de análise e data de validade.

O óleo de soja refinado, utilizado para fins culinários, foi obtido do comércio da cidade do Rio de Janeiro, e se encontrava dentro do prazo de validade.
As características de identidade e qualidade destas amostras foram garantidas através de análises, com seus resultados apresentados na Tabela 1, tópico resultados e discussão, deste trabalho.

\subsection{Amostras provenientes de experimentos de fritura de batatas}

Foram utilizadas, para as análises, vinte amostras provenientes de quatro experimentos, dois para cada tipo de óleo mencionado acima.

Cada experimento foi realizado no laboratório, em condições semelhantes, de acordo com Jorge, et al. (1996), como a seguir: $(100 \pm 0,01) \mathrm{g}$ de batatas frescas descascadas, cortadas em palito, lavadas e secas, foram fritas, em $(550 \pm 0,01) \mathrm{g}$ de óleo em temperaturas iniciais compreendidas entre $(183,1 \pm 1,7){ }^{\circ} \mathrm{C}$, por 3 minutos. Foram utilizadas quatro fritadeiras elétricas domésticas iguais de $1 \mathrm{~L}$, uma para cada experimento, da marca Taurus, Gourmet de Taurus, Barcelona, Espanha, com relação inicial, superfície da fritadeira sobre volume do meio de fritura, de $0,3 \mathrm{~cm}^{-1}$. Cada experimento foi realizado em cinco dias seguidos, e o óleo em cada fritadeira foi submetido por dia a 5 horas de aquecimento, ocorrendo neste período a operação de fritura. A cada dia, ao final de 5 horas de aquecimento, em cada fritadeira, uma alíquota do óleo (amostra) foi coletada para análise. Assim, foram coletadas a cada dia quatro amostras, duas de cada tipo de óleo, provenientes da fritura e aquecidas por 5, 10, 15, 20 e 25 horas, respectivamente. Em cada experimento, não houve reposição ou substituição do óleo.

Cada amostra obtida por dia foi estocada à temperatura de $-20^{\circ} \mathrm{C}$, até o momento das análises.

\subsection{Reagentes e padrões}

Foram utilizados solventes e reagentes de grau P. A. (para análise), com exceção dos solventes, hexano e tetrahidrofurano, para as análises cromatográficas que foram de grau para análise de resíduos.

Os padrões de ésteres metílicos de ácidos graxos foram obtidos da Sigma- Aldrich Chemie GmbH (Steinhein, Germany).

\subsection{Métodos analíticos}

As seguintes determinações foram efetuadas, em duplicata, de acordo com o método da International Union of Pure and Applied Chemistry (IUPAC, 1992): acidez em ácido oléico, $\%, \mathrm{~m} / \mathrm{m}$, método 2.201 ; índice de peróxidos, meq $\mathrm{O}_{2} / \mathrm{kg}$, método 2.501; matéria insaponificável, $\%, \mathrm{~m} / \mathrm{m}$, método 2.401 . O primeiro e o segundo método utilizam a técnica de volumetria; o terceiro método utiliza extração por solvente orgânico e gravimetria (IUPAC, 1992).

A composição percentual, $\mathrm{m} / \mathrm{m}$, da fração dos ácidos graxos foi realizada, em todas as amostras do estudo, através da análise cromatográfica a gás dos ésteres metílicos, destes ácidos, obtidos a partir da dissolução de $(50 \pm 0,0001) \mathrm{mg}$ da amostra em $1 \mathrm{~mL}$ de hexano e posterior derivação com adição 
de duas gotas da solução de $\mathrm{KOH} 2 \mathrm{~N}$ em metanol, de acordo com os métodos 2.301 e 2.302 da IUPAC (1992). Utilizou-se um cromatógrafo da Hewlett-Packard, modelo 6890, Avondale, PA, USA, equipado com detector por ionização em chama; injetor automático marca Agilent Technologies, Karlsruhe, Germany, com divisão de fluxo; e coluna cromatográfica, de alta resolução de sílica fundida, da Hewlett-Packard, Avondale, PA, USA, com fase estacionária de poli (etileno glicol), nas dimensões de $60 \mathrm{~m}$ de comprimento; 0,25 $\mathrm{mm}$ de diâmetro interno e $0,25 \mu \mathrm{m}$ de espessura de fase estacionária. As seguintes condições cromatográficas foram adotadas: temperatura da coluna: $200^{\circ} \mathrm{C}(2$ minutos $) / 2{ }^{\circ} \mathrm{C} /$ minuto $/ 240^{\circ} \mathrm{C}$ (30 minutos); temperatura do detector e do injetor: $250^{\circ} \mathrm{C}$; fluxo do gás carreador $\left(\mathrm{N}_{2}\right): 1,0 \mathrm{~mL} /$ minuto; razão de divisão de fluxo: 40,0:1; fluxo do gás auxiliar $\left(\mathrm{N}_{2}\right): 45,0 \mathrm{~mL} /$ minuto; fluxo do $\mathrm{H}_{2}: 40,0 \mathrm{~mL} /$ minuto; fluxo do ar sintético: $450,0 \mathrm{~mL} /$ minuto; volume injetado da amostra: $1,0 \mu \mathrm{L}$.

A identificação dos picos realizou-se através da comparação dos tempos de retenção dos padrões dos ésteres metílicos dos ácidos graxos de interesse.

A composição percentual de ácidos graxos presentes em cada amostra foi obtida a partir da área dos picos cromatográficos, do cromatograma, de cada ácido graxo em relação à soma das áreas de todos os ácidos graxos da amostra.

A determinação quantitativa dos triacilgliceróis polimerizados (polímeros) foi obtida em todas as amostras do estudo, de acordo com o método 2.508 da IUPAC (1992), como a seguir: $(50 \pm 0,0001) \mathrm{mg}$ da amostra foram dissolvidos em $1 \mathrm{~mL}$ de tetrahidrofurano, a seguir foram submetidos à análise cromatográfica a líquido de exclusão por tamanho de partículas. Foram utilizados: um injetor Rheodyne $7725 \mathrm{i}$ com loop de $20 \mu \mathrm{L}$; uma bomba Waters 510; duas colunas cromatográficas, conectadas em série, de 100 e $500 \AA$, com dimensões de $30 \mathrm{~cm}$ de comprimento e $0,77 \mathrm{~cm}$ de diâmetro interno, cada uma, empacotada com copolímero, com ligações cruzadas, de estireno divinilbenzeno, com tamanho de partícula de $5 \mu \mathrm{m}$, da Waters Associates; e detector de índice de refração modelo L-7490 da Merck-La Chrom. Foram injetados, em condições isocráticas, $10 \mu \mathrm{L}$ da amostra em um fluxo de $1,5 \mathrm{~mL} /$ minuto de tetrahidrofurano utilizado como fase móvel.

A identificação dos picos dos polímeros foi realizada através da comparação do cromatograma obtido com o perfil cromatográfico padronizado pelo método analítico utilizado.

O teor, em porcentagem, de polímeros foi calculado pela razão entre a soma das áreas dos picos de interesse e a soma das áreas de todos os picos apresentados, multiplicada por 100.

\subsection{Análises estatísticas}

Foi utilizado o programa da Microsoft Excel para o tratamento dos dados.

Foram avaliadas as correlações entre os valores de formação de polímeros e de perda de ácidos graxos obtidos nos quatro experimentos de fritura.

\section{Resultados e discussão}

\subsection{Análise das amostras iniciais}

A Tabela 1 apresenta as características de qualidade e identidade dos óleos de palma e de soja. Os resultados das análises demonstraram que as amostras estavam adequadas para serem utilizadas no estudo, pois os parâmetros avaliados estavam dentro dos valores estabelecidos pela legislação brasileira (BRASIL, 2005), ou seja, sua identidade e qualidade estavam garantidas.

\subsection{Análises das amostras provenientes dos experimentos de fritura}

Foram obtidas diferenças significativas nos valores dos teores de polímeros e de ácidos graxos (Tabelas 2 e 3 e Tabelas 4 e 5 , respectivamente), entre cada par de experimentos (utilização do mesmo tipo de óleo), em condições semelhantes e com o mesmo tempo de aquecimento. Estas diferenças são esperadas, pois apesar da utilização do mesmo tipo de óleo e mesma quantidade inicial deste, como também da mesma quantidade de batata e o mesmo tempo de fritura, não se utilizou a mesma fritadeira, nem as mesmas temperaturas iniciais, consideradas como temperaturas utilizadas antes da adição do alimento, e a quantidade do óleo ao final de cada dia em cada fritadeira não foi igual.

Tabela 1. Características de qualidade e identidade dos óleos de palma e de soja.

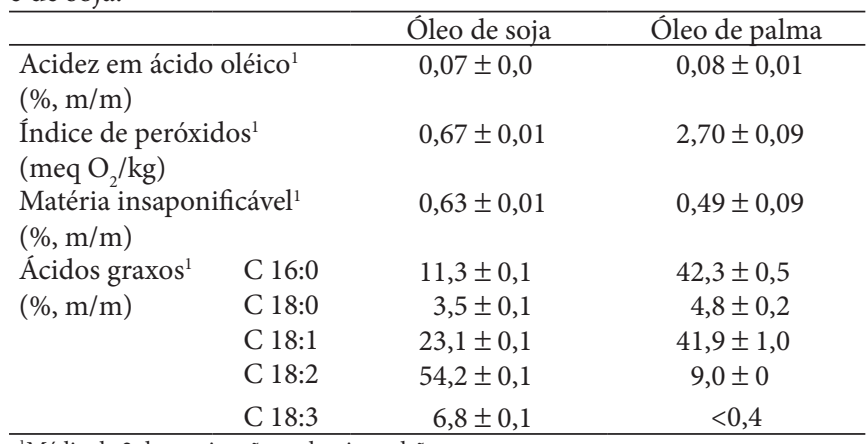

${ }^{1}$ Média de 2 determinações e desvio padrão.

Tabela 2. Resultados analíticos da determinação de polímeros em amostras de óleo de palma, antes da utilização no experimento (inicial) e provenientes de frituras descontínuas de batatas.

\begin{tabular}{|c|c|c|c|c|c|c|c|c|c|c|}
\hline Amostras $^{1}$ & P0 & $\begin{array}{l}\text { P5 } \\
(1)\end{array}$ & $\begin{array}{l}\text { P10 } \\
(1)\end{array}$ & $\begin{array}{cc}\text { P15 } & \text { P20 } \\
(1) & (1)\end{array}$ & $\begin{array}{l}\mathrm{P} 25 \\
(1)\end{array}$ & $\begin{array}{l}\text { P5 } \\
(2)\end{array}$ & $\begin{array}{l}\text { P10 } \\
(2)\end{array}$ & $\begin{array}{l}\mathrm{P} 15 \\
(2)\end{array}$ & $\begin{array}{c}\text { P20 } \\
(2)\end{array}$ & $\begin{array}{l}\text { P25 } \\
(2)\end{array}$ \\
\hline $\begin{array}{l}\text { Polímeros } \\
(\%, \mathrm{~m} / \mathrm{m})\end{array}$ & 0,4 & 1,1 & 2,4 & $6,3 \quad 12,2$ & 19,0 & 2,7 & 8,0 & 13,6 & 18,6 & 23,7 \\
\hline
\end{tabular}

Tabela 3. Resultados analíticos da determinação de polímeros em amostras de óleo de soja, antes da utilização no experimento (inicial) e provenientes de frituras descontínuas de batatas.

\begin{tabular}{lccccccccccc}
\hline Amostras $^{1}$ & S0 & $\begin{array}{c}\text { S5 } \\
(1)\end{array}$ & $\begin{array}{c}\text { S10 } \\
(1)\end{array}$ & $\begin{array}{c}\text { S15 } \\
(1)\end{array}$ & $\begin{array}{c}\text { S20 } \\
(1)\end{array}$ & $\begin{array}{c}\text { S25 } \\
(1)\end{array}$ & $\begin{array}{c}\text { S5 } \\
(2)\end{array}$ & $\begin{array}{c}\text { S10 } \\
(2)\end{array}$ & $\begin{array}{c}\text { S15 } \\
(2)\end{array}$ & $\begin{array}{c}\text { S20 } \\
(2)\end{array}$ & $\begin{array}{c}\text { S25 } \\
(2)\end{array}$ \\
\hline $\begin{array}{l}\text { Polímeros } \\
(\%, 5\end{array}$ & 2,0 & 3,8 & 7,3 & 8,8 & 12,0 & 4,2 & 10,6 & 16,9 & 23,6 & 30,7 \\
& \\
\hline
\end{tabular}


Tabela 4. Composição, g.100 g ${ }^{-1}$ do óleo, dos ácidos graxos em amostras de óleo de palma, antes da utilização no experimento (inicial) e provenientes de experimentos de frituras descontínuas de batatas com o óleo aquecido por distintos períodos de tempo.

\begin{tabular}{|c|c|c|c|c|c|c|}
\hline \multirow{2}{*}{$\begin{array}{c}\text { Tempo de } \\
\text { aquecimento (horas) }\end{array}$} & \multicolumn{5}{|c|}{ Ácidos graxos } & \multirow{2}{*}{$\begin{array}{l}\mathrm{A} \mathrm{G}^{3} \\
(\%)\end{array}$} \\
\hline & C 16:0 & C 18:0 & C 18:1 & C18:2 & Outros $^{2}$ & \\
\hline $0^{1}$ & $42,3 \pm 0,5$ & $4,8 \pm 0,2$ & $41,9 \pm 1,0$ & $9,0 \pm 0$ & $2,0 \pm 0,3$ & 100 \\
\hline 5 & 42,3 & 4,7 & 41,8 & 8,8 & 2,2 & 99,8 \\
\hline 10 & 42,3 & 5,0 & 41,6 & 8,2 & 2,2 & 99,3 \\
\hline 15 & 42,3 & 4,8 & 41,7 & 7,2 & 2,6 & 98,6 \\
\hline \multicolumn{7}{|c|}{$2^{\circ}$ experimento } \\
\hline 5 & 42,3 & 4,7 & 41,8 & 8,2 & 1,8 & 98,8 \\
\hline 10 & 42,3 & 4,6 & 40,9 & 6,6 & 1,9 & 96,3 \\
\hline 15 & 42,3 & 4,8 & 38,8 & 5,5 & 2,6 & 94,0 \\
\hline 20 & 42,3 & 4,7 & 37,2 & 4,2 & 1,8 & 90,2 \\
\hline
\end{tabular}

${ }^{1}$ Média de 2 determinações e desvio padrão da amostra inicial; ${ }^{2}$ menor que $0,4 \%$; ${ }^{3}$ porcentagem total de ácidos graxos.

Tabela 5. Composição, g.100 g $\mathrm{g}^{-1}$ do óleo, dos ácidos graxos em amostras de óleo de soja, antes da utilização no experimento (inicial) e provenientes de experimentos de frituras descontínuas de batatas com óleo aquecido por distintos períodos de tempo.

\begin{tabular}{|c|c|c|c|c|c|c|c|}
\hline \multirow{2}{*}{$\begin{array}{c}\text { Tempo de } \\
\text { aquecimento (horas) }\end{array}$} & \multicolumn{6}{|c|}{ Ácidos graxos } & \multirow{2}{*}{$\begin{array}{c}\mathrm{A} \mathrm{G}^{3} \\
(\%)\end{array}$} \\
\hline & C 16:0 & C 18:0 & C 18:1 & C18:2 & C18:3 & Outros $^{2}$ & \\
\hline $0^{1}$ & $11,3 \pm 0,1$ & $3,5 \pm 0,1$ & $23,1 \pm 0$ & $54,2 \pm 0,2$ & $6,8 \pm 0,1$ & $1,1 \pm 0,1$ & 100 \\
\hline \multicolumn{8}{|c|}{$1^{\circ}$ experimento } \\
\hline 5 & 11,3 & 3,5 & 22,8 & 52,9 & 6,7 & 1,1 & 98,3 \\
\hline 10 & 11,3 & 3,5 & 22,4 & 51,0 & 6,2 & 1,2 & 95,6 \\
\hline 15 & 11,3 & 3,5 & 22,2 & 49,5 & 6,0 & 0,9 & 93,4 \\
\hline 25 & 11,3 & 3,4 & 22,1 & 46,7 & 5,3 & 1,4 & 90,2 \\
\hline \multicolumn{8}{|c|}{$2^{\circ}$ experimento } \\
\hline 5 & 11,3 & 3,4 & 22,8 & 52,3 & 6,4 & 1,2 & 97,4 \\
\hline 10 & 11,3 & 3,5 & 22,4 & 49,5 & 5,6 & 1,1 & 93,4 \\
\hline 15 & 11,3 & 3,4 & 22,1 & 46,6 & 5,2 & 1,1 & 89,7 \\
\hline 20 & 11,3 & 3,4 & 21,7 & 43,6 & 4,6 & 1,0 & 85,6 \\
\hline 25 & 11,3 & 3,5 & 21,6 & 40,7 & 4,1 & 1,2 & 82,4 \\
\hline
\end{tabular}

${ }^{1}$ Média de 2 determinações e desvio padrão da amostra inicial; ${ }^{2}$ menor que $0,4 \%$; e ${ }^{3}$ porcentagem total de ácidos graxos.

A não utilização da mesma fritadeira, por poder acarretar períodos desiguais de aquecimento e resfriamento devido ao sensor de cada fritadeira, e a utilização de temperaturas iniciais diferentes resultam em temperaturas desiguais do óleo em diversos períodos do processo, (antes da adição do alimento, após a adição do alimento, durante a fritura, no final da fritura), no mesmo tempo de aquecimento, ocasionando níveis diferentes de alteração do óleo.

Com relação à quantidade do óleo, este diminui em valores diferentes em cada fritura como conseqüência de quantidades desiguais de óleo absorvidas pelas batatas. Esta absorção podese explicar, dentre outros motivos, pela variação nos teores de umidade da batata, ainda que estas sejam do mesmo lote, como também por valores diferentes de temperatura do óleo antes e durante a fritura. Neste caso, a quantidade final do óleo será desigual a cada dia no mesmo experimento e também em cada par de experimentos, ocasionando diferentes valores de relação final de superfície da fritadeira sobre volume do óleo. Como conseqüência, teremos diferentes níveis de alteração do óleo.
Estas considerações, explicam em parte, porque o processo de fritura é difícil de ser controlado. No entanto, para este estudo não foi necessário um processo controlado.

Nas Tabelas 2 e 3 estão os resultados analíticos da determinação de polímeros em todas as amostras dos óleos de palma e de soja, respectivamente. Em cada tabela, a amostra de número "zero" é a inicial e as de número 5 a 25 , são as provenientes dos dois experimentos de fritura de batatas, (1) e (2), com o óleo aquecido por $5 ; 10 ; 15 ; 20$ e 25 horas, respectivamente, como mencionado na parte de material e métodos.

Verificou-se nas duas tabelas, que os polímeros foram formados e que este resultado já era esperado uma vez que a dimerização e polimerização dos ácidos graxos ocorrem fundamentalmente na presença destes ácidos insaturados, mono e poliinsaturados, na ausência de oxigênio a altas temperaturas e na presença de oxigênio a baixas e altas temperaturas (COWAN, 1962; FORMO et al., 1979; FRANKEL, 1991; FRANKEL, 1998; JORGE, 1996; PASCHKE et al., 1964; WHEELER; MILUN; 
LINN, 1970), e estas condições se apresentaram no estudo. E também estudos têm demonstrado que óleos e gorduras em presença principalmente de altas temperaturas e ar sofrem alteração com formação de um número considerável de substâncias dentre elas os dímeros e polímeros de ácidos graxos (CHANG; PETERSON; HO, 1978; DOBARGANES; PÉREZ-CAMINO, MÁRQUEZ-RUÍZ 1998; FRITSCH, 1981; GASPAROLI, 1998; JORGE et al., 1996; MÁRQUEZ-RUÍZ; TASIOULA-MARGARI; DOBARGANES, 1995). Também se verificou que houve crescente aumento no teor destas substâncias com o aumento do tempo de aquecimento.

As Tabelas 4 e 5 mostram a composição real, expressa em g. $100 \mathrm{~g}^{-1}$ do óleo, dos ácidos graxos das amostras dos óleos de palma e de soja, respectivamente. A última coluna de cada tabela indica a porcentagem total de ácidos graxos em $100 \mathrm{~g}$ do óleo.

Os valores obtidos, apresentados nas referidas tabelas, para os teores dos ácidos graxos insaturados, nos quatro experimentos nas condições do estudo demonstraram, como esperado, a crescente diminuição da concentração dos ácidos graxos insaturados com o aumento do tempo de aquecimento, uma vez que estas substâncias são termoxidadas na presença principalmente de altas temperaturas e ar (CHANG; PETERSON; HO, 1978; DOBARGANES; PÉREZ-CAMINO; MÁRQUEZ-RUÍZ, 1988; GASPAROLI, 1998; FRITSCH, 1981; JORGE et al., 1996; MÁRQUEZ-RUÍZ; TASIOULA-MARGARI; DOBARGANES, 1995; NAWAR, 1998; SCHWARTZ; RADY; CASTAÑEDA, 1994; VELASCO et al., 2004a; VELASCO et al., 2004b).

Como os ácidos graxos saturados não sofrem prioritariamente alteração, para se obter a composição real, expressa em g. $100 \mathrm{~g}^{-1}$ do óleo, a partir da composição porcentual, da fração dos ácidos graxos, considerou-se que a concentração do ácido saturado majoritário (C16:0) não se altera, ao longo da fritura, permanecendo com a concentração igual à concentração das amostras iniciais, ou seja, 42,3 e 11,3\%, para os óleos de palma e de soja, respectivamente. Então, as porcentagens dos outros ácidos graxos foram obtidas por normalização em relação à concentração inicial do ácido palmítico (DOBARGANES; PÉREZ-CAMINO; MÁRQUEZ-RUÍZ, 1988).

Observa-se, nestas tabelas, que as porcentagens do outro ácido saturado (C18:0) se mantêm com valores próximos aos valores de cada amostra inicial, por isso foram aplicados cálculos matemáticos para se deduzir a perda de cada ácido diretamente dos valores da tabela.

As perdas expressas em porcentagens, sobre a quantidade inicial dos principais ácidos graxos insaturados no óleo de pal$\mathrm{ma}$, ao final do primeiro e do segundo experimento de fritura foram, respectivamente, 47,8 e 62,2 , para o ácido linoléico e 10,3 e 15,0, para o ácido oléico. Como esperado, a perda destes ácidos graxos aumentou com o seu grau de insaturação.

As perdas expressas em porcentagens, sobre a quantidade inicial dos principais ácidos graxos insaturados no óleo de soja, ao final do primeiro e do segundo experimento de fritura foram, respectivamente, 39,7 e 22,1, para o ácido linolênico; 24,9 e 13,8, para o ácido linoléico e 6,5 e 4,3, para o ácido oléico. Da mesma forma, como no óleo de palma, como esperado, a perda dos ácidos graxos aumentou com o seu grau de insaturação.
As Figuras 1 e 2 ilustram a correlação linear entre o percentual de perda dos principais ácidos graxos insaturados e a formação de polímeros nos experimentos de fritura utilizando os óleos de palma e de soja, respectivamente.

Os coeficientes de correlação obtidos demonstraram a excelente relação entre a formação de polímeros e a perda por degradação dos ácidos graxos insaturados.

A Figura 3 ilustra as correlações lineares entre a perda dos principais ácidos graxos insaturados e o teor de polímeros, nos experimentos de fritura de batatas utilizando os óleos de palma e de soja.

Como se podem observar, os valores das ordenadas na origem $(82,7$ e 51,4$)$ são similares aos valores iniciais do total de ácidos insaturados $(84,1$ e 50,9) para os óleos de soja e palma, respectivamente, com conteúdo muito baixo em polímeros, $\mathrm{o}$ que indica a excelência do ajuste linear.

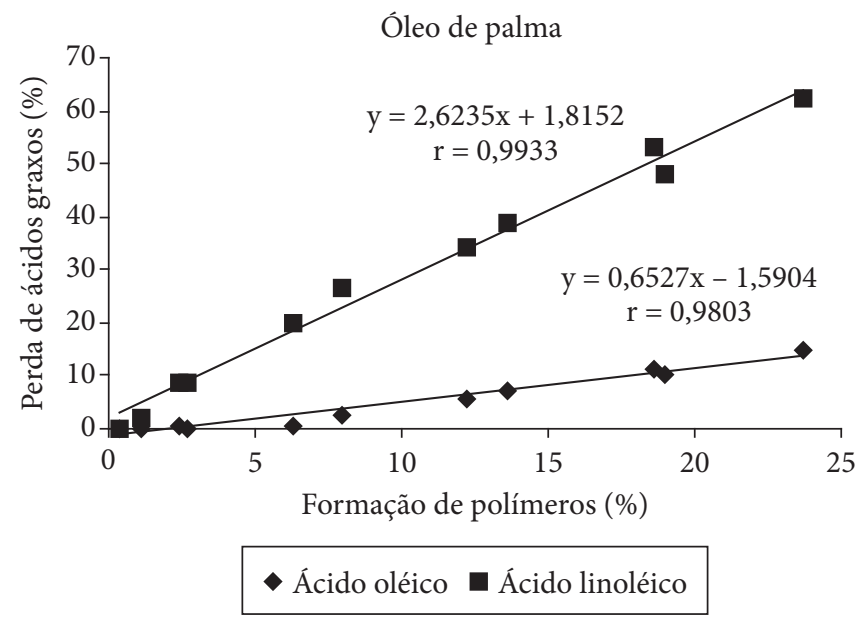

Figura 1. Percentual de perda dos principais ácidos graxos insaturados na composição de ácidos graxos em relação à formação de polímeros, nos experimentos de fritura de batatas utilizando o óleo de palma.

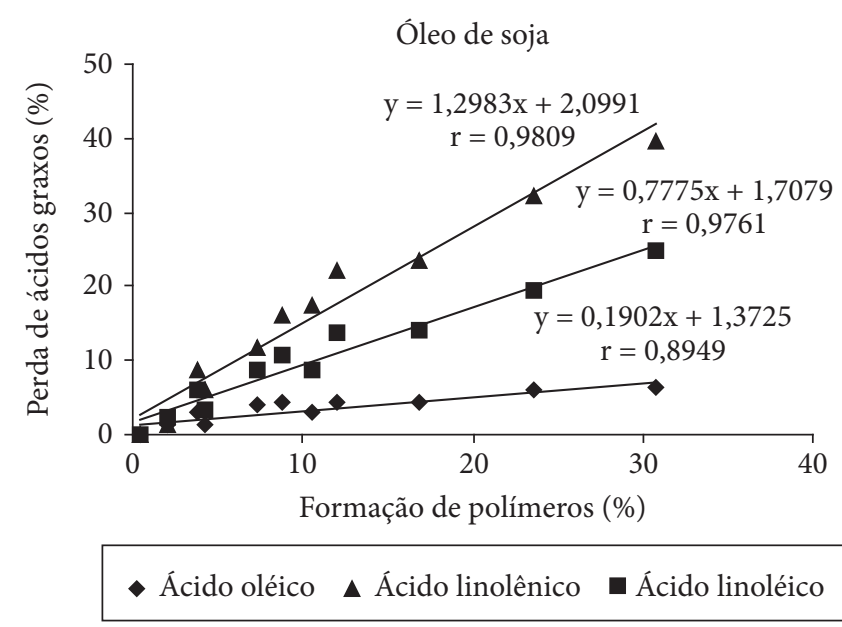

Figura 2. Percentual de perda dos principais ácidos graxos insaturados em relação à formação de polímeros, nos experimentos de fritura de batatas utilizando o óleo de soja. 


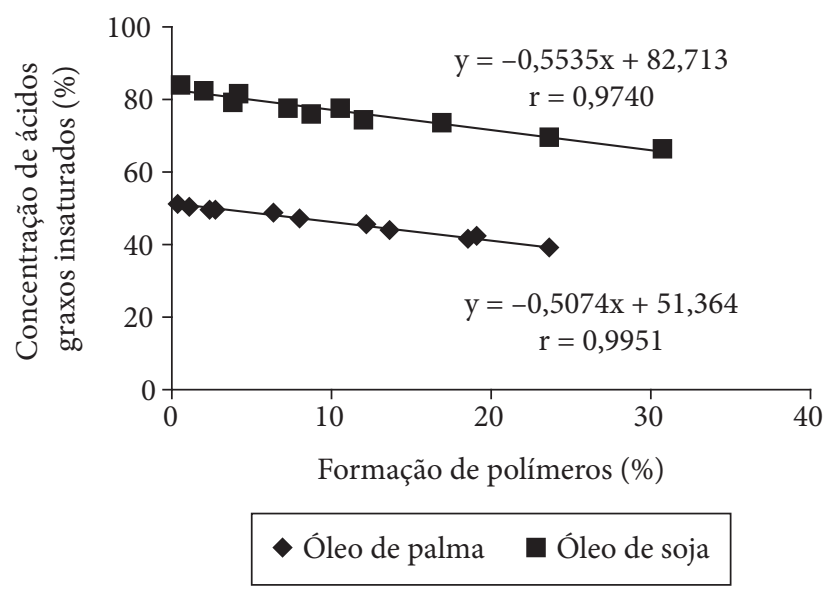

Figura 3. Concentração dos principais ácidos graxos insaturados em relação à formação de polímeros, nos experimentos de fritura de batatas utilizando os óleos de palma e de soja.

Os altos coeficientes de correlação obtidos, 0,9951 e 0,9740, para os óleos de palma e de soja, respectivamente, demonstraram que a alteração, destes óleos submetidos a frituras descontínuas de batatas, pode ser eficazmente verificada através da determinação quantitativa de polímeros. Estes dados confirmam a determinação de polímeros como mais um parâmetro de verificação da alteração destes óleos em frituras de batatas, além do parâmetro mais estabelecido pelas leis internacionais para uso de óleos e gorduras em frituras, que é a porcentagem de compostos polares (DANA; SAM SAGUY, 2001).

\section{Conclusões}

Os dados obtidos dos experimentos de frituras descontínuas de batatas utilizando os óleos refinados de palma e de soja, nas condições do estudo, demonstraram que:

- maiores graus de alteração ocorreram nos ácidos graxos mais insaturados; e

- a alteração destes óleos pode ser eficazmente verificada através da determinação quantitativa de polímeros, dada a sua excelente correlação com a perda de ácidos graxos insaturados.

\section{Agradecimentos}

Eliana Rodrigues Machado agradece à Coordenação de Aperfeiçoamento de Pessoal de Nível Superior (CAPES), pela bolsa de estágio de doutorando recebida; e ao "Instituto de la Grasa" do "Consejo Superior de Investigaciones Científicas", Sevilha, Espanha, onde o trabalho foi realizado.

Os autores agradecem à Sra. Mercedes Gimenez pela assistência; e ao Grupo Agropalma pela doação do óleo de palma.

\section{Referências bibliográficas}

BERDEAUX, O.; MÁRQUEZ-RUÍZ, G.; DOBARGANES, M. C. Characterization, quantitation and evolution of monoepoxy compounds formed in model systems of fatty acid methyl esters and monoacid triglycerides heated at high temperature. Grasas y Aceites, v. 50, n. 1, p. 53-59, 1999.

BRASIL. ANVISA. Agência Nacional de Vigilância Sanitária. Resolução RDC n 270, de 22 de setembro de 2005. Aprova o "Regulamento Técnico para óleos vegetais, gorduras vegetais e creme vegetal". Diário Oficial da República Federativa do Brasil, Brasília, DF, 23 set. 2005. Disponível em: <http://www.anvisa.gov.br/e-legis/> Acesso em: 14 Maio 2006.

CHANG, S. S.; PETERSON, R. J.; HO, C. Chemical reactions involved in the deep-fat frying of foods. Journal of the American Oil Chemists' Society, v. 55, n. 10, p. 718-727, 1978.

COWAN, J. C. Dimer acids. Journal of the American Oil Chemists' Society, v. 39, n. 12, p. 534-545, 1962.

CROON, L. B., et al. Comparative study of analytical methods for quality evaluation or frying fat. Fette, Seifen, Anstrichmittel, v. 88, n. 3, p. 87-91, 1986.

DANA, D.; SAM SAGUY, I. Frying of nutritious foods: obstacles and feasibility. Food Science Technology Research, v. 7, n. 4, p. 265-279, 2001.

DOBARGANES, M. C.; MÁRQUEZ-RUÍZ, G. Calidad de las grasas de fritura en el sector de restauración de Andalucía. Grasas y Aceites, v. 46, n. 2, p. 115-120, 1995.

Oxidized fats in foods. Current Opinion in Clinical Nutrition and Metabolic Care, v. 6, n. 2, p. 157-163, 2003.

Regulation of used frying fats and validity of quick tests for discarding the fats. Grasas y Aceites, v. 49, n. 3-4, p. 331-335, 1998.

DOBARGANES, M. C.; PÉREZ-CAMINO, M. C.; MÁRQUEZ-RUÍZ, G. Fatty acid composition: a useful tool for the determination of alteration level in heated fats. Revue Française des Corps Gras, v. 35, n. 2, p. 67-70, 1988.

High performance size exclusion chromatography of polar compounds in heated and non-heated fats. Fat Science and Technology, v. 90, n. 8, p. 308-311,1998.

FORMO, M. V. et al. Bailey's Industrial Oil and Fatt Products. 4 ed. New York: J. Wiley \& Sons, 1979. 839 p.

FRANKEL, E. N. Review. Recents advances in lipid oxidation. Journal of the Science of Food and Agriculture, v. 54, n. 4, p. 495-511, 1991.

Frying fats. In: Lipid oxidation. Dundee, U.K.: Oil Press, 1998. p. 227-248.

FRITSCH, C. W. Measurements of frying fat deterioration: a brief review. Journal of the American Oil Chemists' Society, v. 58, n. 3, p. 272-274, 1981.

GASPAROLI, A. The formation of new compounds. Grasas y Aceites, v. 49, n. 3-4, p. 303-309, 1998.

GERTZ, C. Chromatographiche methoden bei der untersuchung von fritierfetten. Fette, Seifen, Anstrichmittel, v. 88, n. 12, p. 475-488, 1986.

IUPAC - International Union of Pure and Applied Chemistry. Standard methods for the analysis of oils, fats and derivatives. 7 ed. Oxford: Pergamon Press, 1992, supl. 1., 1 v.

JORGE, N. Estudo do comportamento do óleo de girassol e do efeito do dimetil polisiloxano em termoxidação e frituras. Campinas, 1996. Tese - (Doutorado em Tecnologia de Alimentos), Universidade de Campinas - UNICAMP.

JORGE, N., et al. Influence of Dimethylpolysiloxane Addition to Edible Oils: Performance of Sunflower Oil in Discontinuous and 
Continuous Laboratory Frying. Grasas y Aceites, v. 47, n. 1-2, p. 20-25, 1996.

LAKE, R. J.; SCHOLES, P. Quality and consumption of oxidized lipids from deep-frying fats and oils in New Zealand. Journal of the American Oil Chemists' Society, v. 74, n. 9, p. 1065-1068, 1997.

MÁRQUEZ-RUIZ, G.; TASIOULA-MARGARI, M.; DOBARGANES, M. C. Quantitation and distribution of altered fatty acids in frying fats. Journal of the American Oil Chemists' Society, v. 72, n. 10, p. 1171-1176, 1995.

MATTOS, E. S.; ANS, V. G.; JORGE, N. Utilização do Kit Oil Test para Avaliação da Alteração dos Óleos de Fritura. Higiene Alimentar, v. 11, n. 75, p. 40-47, 2000.

NAWAR, W. W. Volatile components of the frying process. Grasas y Aceites, v. 49, n. 3-4, p. 271-274, 1998.

PASCHKE, R. F. et al. Dimer acid structures. The dehydro-dimer from methyl oleate and di-t-butyl peroxide. Journal of the American Oil Chemists' Society, v. 41, n. 1, p. 56-60, 1964.

SCHWARTZ, D. P.; RADY, A. H.; CASTAÑEDA, S. The formation of oxo- and hydroxy-fatty acids in heated fats and oils. Journal of the American Oil Chemists' Society, v. 71, n. 4, p. 441-444, 1994.
SÉBÉDIO, J. L. et al. Etat d'ultération de quelques huiles de friture prélevées en restauration. Revue Française des Corps Gras, v. 34, n. 1, p. 15-18, 1987.

SKRÖKKI, A. Test used for examining the quality of frying oils. Fat Science Technology, v. 97, n. 10, p. 384-386, 1995.

VAHCIC, N.; HRUSKAR, M. Quality and sensory evaluation of used frying oil from restaurants. Food Technology and Biotechnology, v. 37, n. 2, p. 107-112, 1999.

VELASCO, J. et al. Formation and evolution of monoepoxy fatty acids in thermoxidized olive and sunflower oils and quantitation in used frying Oliz from restaurants and freíd-food outlets. Journal of Agricultural and Food Chemistry, v. 52, n. 14, p. 4438-4443, 2004a.

Formation of short-chain glycerol-bound oxidation products and oxidised monomeric triacylglycerols during deep-frying and occurrence in used frying fats. European Journal of Lipid Science and Technology, v. 106, n. 11, p. 728-735, 2004b.

WHEELER, D. H.; MILUN, A.; LINN, F. Dimmer acid structures: ciclic structures of clay catalyzed dimers of normal linoleic acid, 9-cis, 12-cis-octadecadienoic acid. Journal of the American Oil Chemists' Society, v. 47, n. 7, p. 242-244, 1970. 